\title{
Feasibility of Secondary Follicle Isolation, Culture and Achievement of In-Vitro Oocyte Maturation from Superovulated Ovaries: An Experimental Proof-of-Concept Study Using Mice
}

\author{
Xia Hao ${ }^{1,2}$, Amandine Anastácio ${ }^{1,2}$ and Kenny A. Rodriguez-Wallberg 1,2,3,*(D) \\ 1 Department of Oncology and Pathology, Karolinska Institutet, Solna, SE-171 76 Stockholm, Sweden; \\ xia.hao@ki.se (X.H.); anastacio.amandine@gmail.com (A.A.) \\ 2 Laboratory of Translational Fertility Preservation, BioClinicum, SE-171 64 Stockholm, Sweden \\ 3 Department of Reproductive Medicine, Division of Gynecology and Reproduction, \\ Karolinska University Hospital, Novumhuset Plan 4, SE-141 86 Stockholm, Sweden \\ * Correspondence: kenny.rodriguez-wallberg@ki.se
}

\section{check for}

updates

Citation: Hao, X.; Anastácio, A.; Rodriguez-Wallberg, K.A. Feasibility of Secondary Follicle Isolation, Culture and Achievement of In-Vitro Oocyte Maturation from

Superovulated Ovaries: An Experimental Proof-of-Concept Study Using Mice. J. Clin. Med. 2021, 10, 2757. https://doi.org/10.3390/ jcm10132757

Academic Editor: Alberto Revelli

Received: 10 April 2021

Accepted: 18 June 2021

Published: 23 June 2021

Publisher's Note: MDPI stays neutral with regard to jurisdictional claims in published maps and institutional affiliations.

Copyright: (C) 2021 by the authors. Licensee MDPI, Basel, Switzerland. This article is an open access article distributed under the terms and conditions of the Creative Commons Attribution (CC BY) license (https:// creativecommons.org/licenses/by/ $4.0 /)$.

\begin{abstract}
Fertility preservation through ovarian stimulation, aiming at cryopreserving mature oocytes or embryos, is sometimes unsuccessful. This clinical situation deserves novel approaches to overcome infertility following cancer treatment in patients facing highly gonadotoxic treatment. In this controlled experimental study, we investigated the feasibility of in-vitro culturing secondary follicles isolated from superovulated ovaries of mice recently treated with gonadotropins. The follicle yields of superovulated ovaries were $45.9 \%$ less than in unstimulated controls. Follicles from superovulated ovaries showed faster growth pace during the initial 7 days of culture and secreted more $17 \beta$-estradiol by the end of culture vs controls. Parameters reflecting the outcome of follicular development and oocyte maturation competence in vitro were similar between superovulated and control groups, with a similar follicle size at the end of culture and around $70 \%$ survival. Nearly half of cultured follicles met the criteria for in-vitro maturation in both groups and approximately $60 \%$ of those achieved a mature MII oocyte, similarly in both groups. Over $60 \%$ of obtained MII oocytes displayed normal-looking spindle and chromosome configurations, without significant differences between the groups. Using a validated follicle culture system, we demonstrated the feasibility of secondary follicle isolation, in-vitro culture and oocyte maturation with normal spindle and chromosome configurations obtained from superovulated mice ovaries.
\end{abstract}

Keywords: female fertility preservation; superovulation; follicle isolation; secondary follicle culture; in-vitro maturation; ovarian tissue

\section{Introduction}

Effective cancer screening, early diagnosis and advanced treatment have improved cancer patients' long-term survival rate [1,2]. However, as an off-target effect from radiotherapy and chemotherapy, the development of infertility is negatively impacting cancer survivors' life quality [3]. In adult female patients, cryopreservation of mature oocytes and embryos are well-established fertility preservative methods [4-7]. In this context, an increasing population of women undergo controlled ovarian stimulation (COS) treatment with gonadotropins worldwide, aiming at the retrieval of multiple mature oocytes in a single cycle. The clinical pregnancy rate of using cryopreserved mature oocytes that are fertilized and result in an embryo transfer is around 40\% [8,9]. One cycle of COS treatment typically requires two weeks to be completed. In some cases, a poor ovarian response to hormonal stimulation may be evident, and the women do not gain any frozen oocytes or embryos from the COS treatment. As patients with cancer should not delay initiation of 
oncologic treatment, there is a lack of time to plan for additional COS cycles. Alternative fertility preservative options need to be developed for those cases.

Cryopreservation of ovarian tissue is an alternative method for female fertility preservation [10]. The method requires surgery, usually a minimally invasive laparoscopy that can be planned shortly, and it does not require hormonal stimulation. The success rate of ovarian tissue transplantation is difficult to accurately calculate, as many cases may still remain unreported, however the data provided from a Spanish fertility preservation program reported a $46.7 \%$ spontaneous pregnancy rate [11], while a meta-analysis reported a live birth and ongoing pregnancy rate of $37.7 \%$ [12]. The methods for ovarian tissue cryopreservation provide an effective preservation of small primordial follicles representing the ovarian pool, while the growing follicles including those at the preantral stage do not usually survive after thaw $[13,14]$. Collection of cumulus-oocyte complexes (COCs) and denuded oocytes followed by in-vitro maturation (IVM) to improve fertility preservation options during ovarian tissue cryopreservation have been reported [15-17]. The use of secondary follicles reported in this study as a resource of mature oocytes has not been previously reported.

The study was designed as a proof-of-concept aiming at developing a method to rescue follicles after a failed COS attempt. In such clinical situations, the cryopreservation of ovarian tissue is not recommended, as the ovarian morphology is highly affected, and thus the cryopreservation of the ovarian cortex is unlikely to be successful according to the currently standardized methods [18]. In this study, the feasibility of secondary follicle isolation and in-vitro growth up to mature oocytes from superovulated mice ovaries was investigated. The culture of secondary follicles from unstimulated ovaries and achievement of mature oocytes has been previously described and used over the years [19-21]. In our controlled experimental study, C57BL/6J mice undergoing in-vitro fertilization (IVF) were used. The mice strain has been previously validated and is widely used in basic research on fertility preservation [22-26]. The age of the mouse was $4-5$ weeks to mimic adult women of a young reproductive age. A control group of mice ovaries without hormonal stimulation was used to compare follicle development during in-vitro culture and maturation competence after IVM. Several in-vitro culture outcomes including follicle attachment to culture dish, follicular sizes and growth curve, antrum-like cavity formation, 12 days' survival rate and secreted $17 \beta$-estradiol were evaluated to assess follicular growth. In addition, selected follicles were matured in vitro and oocyte maturation outcomes were evaluated as well as the spindle and chromosome configurations of obtained mature oocytes.

\section{Materials and Methods}

\subsection{Chemicals}

All chemicals used in this study were purchased from Sigma-Aldrich ${ }^{\circledR}$ (St. Louis, MO, USA) or Gibco, Thermofischer Scientific ${ }^{\circledR}$ (Paisley, UK), unless otherwise stated.

\subsection{Animals and Grouping}

For this study, 28 4-5 week old C57BL/6J female mice were used. The mice were included in two groups, either undergoing treatment with gonadotropins and superovulation for IVF at the experimental research facility PKL5, Novum, Karolinska Institutet, Huddinge, $(n=19)$, or used as a control group without stimulation $(n=9)$. The superovulation was induced with an intraperitoneal injection of pregnant mare's serum gonadotrophin (5 IU, Folligon ${ }^{\circledR}$, MSD animal health, Brussel, Belgium) followed by an injection of human chorionic gonadotrophin (hCG, $5 \mathrm{IU}$, Chorulon ${ }^{\circledR}, \mathrm{MSD}$ animal health, Boxmeer, Holland) $50 \mathrm{~h}$ later. Mice were sacrificed $13.5 \mathrm{~h}$ after hCG stimulus for collection of the ovaries and the cumulus-oocyte complexes (COCs). All procedures were performed in three independent trails.

All the experimental procedures described were carried out in agreement with ethics permits (03711-2020 and 1372-17) accorded by Karolinska Institutet's Ethics Committee for research using experimental laboratory animals. 


\subsection{Follicle Isolation and Secondary Follicle Culture}

Ovaries were collected in dissection medium which was Leibovitz-15 medium supplemented with $10 \%$ fetal bovine serum (FBS), $100 \mathrm{IU} / \mathrm{mL}$ of penicillin and $100 \mu \mathrm{g} / \mathrm{mL}$ of streptomycin, and kept at $4{ }^{\circ} \mathrm{C}$, if not used immediately, for a maximum of $6 \mathrm{~h}$.

Secondary follicles were mechanically isolated in a dissection medium using microfine U-100 insulin syringes ( $0.3 \mathrm{~mL}$, BD Medical, Le Pont-de-Claix Cedex, France) under stereo-microscope (Nikon ${ }^{\circledR}$, Tokyo, Japan). Intact follicles with a diameter of 100-130 $\mu \mathrm{m}$, with two or more granulosa layers, with few attached theca cells and with a visible, round and central oocyte were selected for in-vitro culture [19-21]. The selected follicles were cultured individually in culture dishes (TC-Schale 60, Standard, Nümbrecht, Germany) containing $10 \times 10 \mu \mathrm{L}$ culture medium droplets, covered with $5 \mathrm{~mL}$ of mineral oil. The culture medium used was $\alpha$-minimal essential medium ( $\alpha$-MEM) GlutaMAX enriched with $5 \%$ FBS, $5 \mu \mathrm{g} / \mathrm{mL}$ insulin, $10 \mu \mathrm{g} / \mathrm{mL}$ transferrin, $100 \mathrm{mIU} / \mathrm{mL}$ recombinant follicle stimulating hormone (GONAL-F, Merck Europe B.V., Amsterdam, Holland). The follicles were cultured for 12 days in a humidified incubator at $37^{\circ} \mathrm{C}$ and with $5 \% \mathrm{CO}_{2}$. The day the follicles were selected for culture was designated as Day 0 and the last day as Day 12 . On Day 1 of culture, $10 \mu \mathrm{L}$ of culture medium was added to each droplet. Thereafter, half of the medium was refreshed every other day and the follicles were observed under an inverted microscope (Nikon ${ }^{\circledR}$, Tokyo, Japan) for morphological analysis and measurement. Follicle diameter was assessed using a calibrated ocular micrometer, and two perpendicular measures including the granulosa cell mass without the theca cells of each follicle were registered. The collected medium was diluted in $90 \mu \mathrm{L}$ of $\alpha$-MEM with bovine serum albumin (BSA, $40 \mathrm{mg} / \mathrm{mL}$ ) and stored at $-20{ }^{\circ} \mathrm{C}$ for further use. On Day 12 of culture, follicles with a size $\geq 200 \mu \mathrm{m}$, presenting a clear granulosa cell proliferation and a visible round oocyte were classified as growing follicles.

\subsection{Oocyte In-Vitro Maturation}

Follicles with at least $400 \mu \mathrm{m}$ of diameter on day 12 were selected for IVM. Oocytes of selected follicles with some surrounding granulosa cells were individually transferred under stereo-microscope to maturation dishes (TC-Schale 60, Standard, Nümbrecht, Germany) containing $10 \times 20 \mu \mathrm{L}$ maturation medium droplets covered with $5 \mathrm{~mL}$ of mineral oil, then incubated at $37^{\circ} \mathrm{C}$, with $5 \% \mathrm{CO}_{2}$. The maturation medium was culture medium supplemented with $1.5 \mathrm{IU} / \mathrm{mL}$ recombinant $\mathrm{hCG}$ and $5 \mathrm{ng} / \mathrm{mL}$ recombinant epidermal growth factor. Maturation dishes were equilibrated in the incubator overnight before use.

Formation of COCs were verified 16-20 h after incubation and oocyte denudation was performed to evaluate the oocyte maturation status. Oocytes with a visible polar body were classified as mature (Metaphase II, MII) or immature (germinal vesicle, GV) if a germinal vesicle was visible. Oocytes with neither polar body nor germinal vesicle were classified as Metaphase I (MI).

\subsection{Spindle and Chromosome Configuration Analysis}

After IVM, all denuded mature MII oocytes in superovulated and control groups were collected and washed in Dulbecco's Phosphate-Buffered Saline containing $0.1 \%$ polyvinyl alcohol (washing buffer). Thereafter, oocytes were fixed with $2 \%$ formaldehyde in washing buffer containing $0.2 \%$ Triton X-100 for $40 \mathrm{~min}$. After fixation, the oocytes were incubated in blocking buffer (washing buffer supplemented with $1 \%$ BSA) overnight at $4{ }^{\circ} \mathrm{C}$. They were blocked for 40 min with a blocking buffer containing $10 \%$ FBS. Then, oocytes were incubated with mouse monoclonal anti- $\alpha$-tubulin antibody (T9026, 1:1000) in blocking buffer for $45 \mathrm{~min}$ followed by Alexa Fluor 488-labelled goat anti-mouse IgG H\&L (ab150113, Abcam, UK, 1:200) in a blocking buffer for $40 \mathrm{~min}$ at $37^{\circ} \mathrm{C}$. Then, oocytes were incubated with $10 \mu \mathrm{g} / \mathrm{mL}$ propidium iodide (81845) for $20 \mathrm{~min}$. At the end, oocytes were mounted with Prolong Diamond Antifade mountant (P36965, Invitrogen, Eugene, Oregon) between a cover slip and a microscope slide. Slides were kept at $4{ }^{\circ} \mathrm{C}$ until Confocal imaging. 
Labelled tubulin and chromatin were assessed using Nikon Eclipse Ti microscope (Nikon ${ }^{\circledR}$, Tokyo, Japan) equipped with the appropriate filter sets for analyzing Alexa Fluor 488 and propidium iodide with $100 \times$ oil immersion objective. Oocyte images were captured with Andor iXon Ultra and analyzed by NIS-Elements program. Image analyses were performed using ImageJ 1.53c software (National Institutes of Health, Bethesda, MD, USA).

\subsection{Hormone Assay}

Culture medium samples were collected on Day 5, 9 and 12 and used to measure the secretion of $17 \beta$-estradiol using commercially available enzyme-linked immunoassay kit, $17 \beta$-estradiol (ab108667, Abcam, Germany), following the manufacturers' protocols. The limit of sensitivity for $17 \beta$-estradiol was $8.68 \mathrm{pg} / \mathrm{mL}$. For each estimation time point in each group, the culture medium collected from 5 follicles with similar growth features and from which obtained mature oocytes were pooled together to reach the required volume of sample amount for the essay. The measurements were performed in triplicates.

\subsection{Statistical Analysis}

Statistical analyses were performed using the GraphPad Prism 8.4.3 software (San Diego, CA, USA). Comparisons between the superovulated and the control groups regarding follicular sizes, $17 \beta$-estradiol levels on different culture days and the numbers of isolated secondary follicles were compared by t-tests. Comparisons of the proportions of follicle attachments, antrum-like cavity formation, survival and IVM outcomes and percentages of MII oocytes with spindle defects or chromosome misalignment between superovulated and control group were all performed by Chi-square test (two-sided).

\section{Results}

\subsection{Secondary Follicle Isolation, In-Vitro Development and Maturation}

Isolated secondary follicles yield meeting the criteria for in-vitro culture in the superovulated group was nearly half of the yield observed in the control group $(p<0.05$, Table 1$)$ On Day 1 of culture, $78.7 \%$ of follicles in the superovulated group attached to the bottom of the culture dishes and this ratio increased to $93.3 \%$ on Day 3, which was significantly higher compared with the attachment ratio found in the control group ( $42 \%$ on Day 1 and $76.5 \%$ on Day 3). At the end of culture, similar percentages of follicles had survived in both groups (Table 1). Additionally, antrum-like cavities were observed in $8.9 \%$ of the follicles isolated from the superovulated group and $10 \%$ of the follicles from the control group.

Table 1. Comparison of parameters reflecting follicular growth and oocyte maturation competence $\left({ }^{*} p<0.05,{ }^{* *} p<0.01,{ }^{* * *} p<0.001\right)$, MI: metaphase I, MII: metaphase II, GV: germinal vesicle.

\begin{tabular}{|c|c|c|c|c|}
\hline & & Superovulated & Control & $p$ Values \\
\hline \multirow{3}{*}{ Follicle isolation } & Ovaries used (N) & 38 & 18 & \\
\hline & Follicles cultured (N) & 225 & 200 & \\
\hline & $\begin{array}{l}\text { Follicle yield/ovary } \\
\quad(\text { Mean } \pm \text { SD) }\end{array}$ & $6.0 \pm 0.65^{*}$ & $11.1 \pm 2.08$ & 0.0151 \\
\hline \multirow{6}{*}{ Culture outcomes } & & $\mathrm{N}(\%)$ & $\mathrm{N}(\%)$ & \\
\hline & Attachment on Day 1 & $177(78.7 \%) * * *$ & $84(42.0 \%)$ & $<0.0001$ \\
\hline & Attachment on Day 3 & $210(93.3 \%)^{* * *}$ & $153(76.5 \%)$ & $<0.0001$ \\
\hline & Antrum formation & $20(8.9 \%)$ & $20(10.0 \%)$ & 0.6954 \\
\hline & Survived till Day 12 & $155(68.9 \%)$ & $150(75.0 \%)$ & 0.1624 \\
\hline & Put into maturation & $119(52.9 \%)$ & $99(49.5 \%)$ & 0.4854 \\
\hline \multirow{3}{*}{ Maturation outcomes } & MII oocytes & $76(63.9 \%)$ & $59(59.6 \%)$ & 0.5180 \\
\hline & MI oocytes & $28(23.5 \%)^{* *}$ & $10(10.1 \%)$ & 0.0093 \\
\hline & GV oocytes & $13(10.9 \%)^{* *}$ & $28(28.3 \%)$ & 0.0011 \\
\hline
\end{tabular}

On Day 12, a similar proportion of follicles in each group fulfilled the criteria to undergo IVM and similar percentages of mature MII oocytes were obtained in superovulated $(63.9 \%)$ and control $(59.6 \%)$ groups $(p=0.5180)$ (Table 1$)$. 
The growth behavior and trend during culture displayed by growth curves were similar between the groups of follicles obtained from superovulated and control ovaries, Figure 1a. However, follicles in the superovulated group grew significantly larger than those in the control group between Day 1 and Day 7, while since Day 9, follicles in both groups reached similar mean follicular sizes (Table 2).

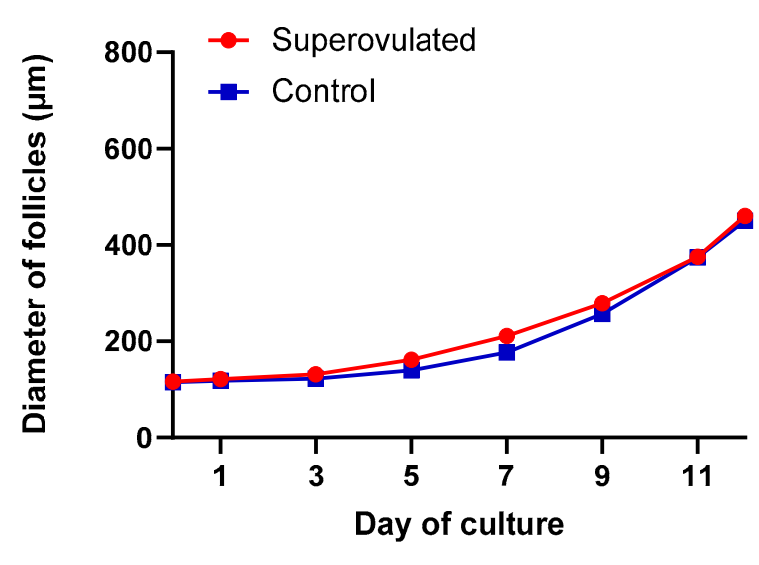

(a)

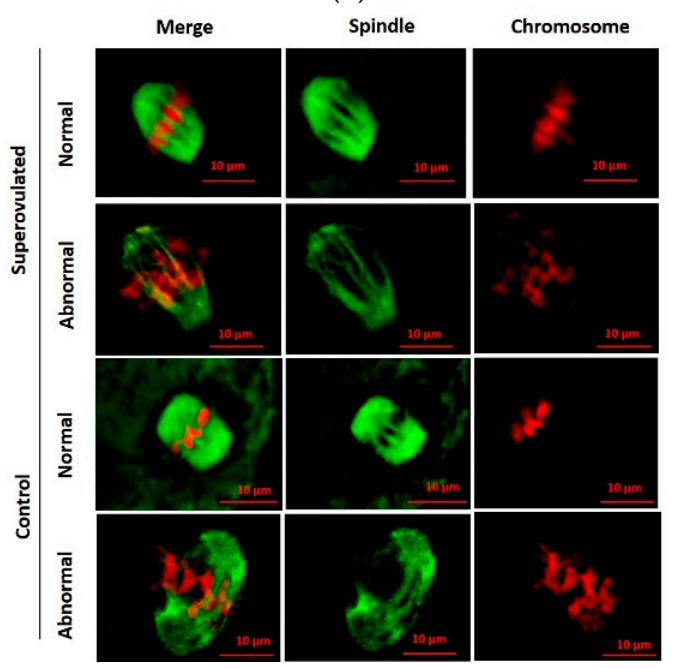

(c)

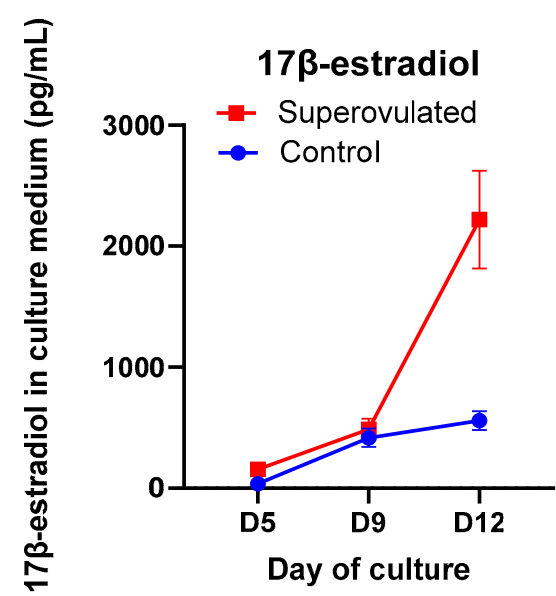

(b)

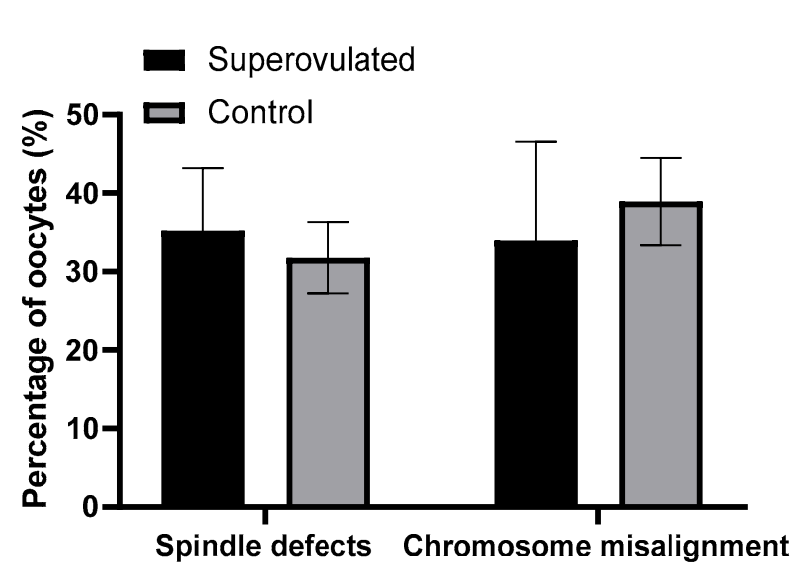

(d)

Figure 1. (a) Follicular growth curves during in-vitro culture; (b) $17 \beta$-estradiol secreted by individual follicles in the culture medium; (c) Representative images showing a normal or abnormal morphology of spindle and chromosome configurations in MII oocytes from control and superovulated groups after IVM. Spindle fibers (green) were detected by immunofluorescence for a-tubulin, while DNA (red) was stained with propidium iodide. Scale bar, $10 \mu \mathrm{m}$; (d) Percentages of MII oocytes with spindle defects or chromosome misalignment in control and superovulated groups.

Table 2. Follicular sizes during culture (mean follicle diameter \pm standard deviation, $\mu \mathrm{m}$ ) of follicles from superovulated ovaries vs. controls $\left({ }^{* *} p<0.01{ }^{* * *} p<0.001\right)$.

\begin{tabular}{|c|c|c|c|c|c|c|c|c|}
\hline & Day 0 & Day 1 & Day 3 & Day 5 & Day 7 & Day 9 & Day 11 & Day 12 \\
\hline Superovulated & $116.6 \pm 9.1$ & $121.6 \pm 11.0 * *$ & $131.6 \pm 21.4^{* * *}$ & $161.5 \pm 46.7^{* * *}$ & $211.2 \pm 84.9^{* * *}$ & $279.0 \pm 134.6$ & $375.5 \pm 157.3$ & $460.1 \pm 150.1$ \\
\hline Control & $115.3 \pm 10.0$ & $118.5 \pm 10.3$ & $122.3 \pm 13.4$ & $139.4 \pm 26.1$ & $177.1 \pm 55.8$ & $256.7 \pm 119.5$ & $374.3 \pm 159.2$ & $450.1 \pm 178.6$ \\
\hline
\end{tabular}




\subsection{Secretion of $17 \beta$-Estradiol during Culture, Spindle and Chromosome Analysis in Mature Oocytes}

Follicle secretion of $17 \beta$-estradiol determined in culture media slightly increased and reached a similar level in the first 9 days of culture in superovulated and control groups, thereafter till Day 12, the level of $17 \beta$-estradiol markedly increased (by $1733.8 \mathrm{pg} / \mathrm{mL}$ ) in the superovulated group, whereas the increase was smaller in control group (by $142.7 \mathrm{pg} / \mathrm{mL}$ ), Figure $1 \mathrm{~b}$. On Day 12, the level of $17 \beta$-estradiol in the superovulated group was significantly higher than control $(p<0.05)$.

MII oocytes with normal- and abnormal-looking spindle and chromosome structures were observed in both groups, Figure 1c. The normal-looking MII oocytes displayed bipolar barrel-shaped spindles and well-aligned chromosomes on the metaphase equator, whereas abnormal-looking MII oocytes showed spindle defects and/or chromosome misalignment. About $30-40 \%$ of MII oocytes presented with chromosome misalignment or spindle defects, and the proportions of abnormal-looking MII oocytes were similar between the superovulated and control groups, Figure $1 \mathrm{~d}$.

\section{Discussion}

In this study, we used ovaries of mice recently superovulated to experimentally investigate the feasibility to obtain secondary follicles in this specific condition. The efficacy of this approach was also investigated by comparing the outcomes with those of follicles retrieved from unstimulated control ovaries. Outcomes of follicles isolated from superovulated ovaries undergoing culture and maturation in vitro indicated the feasibility of this approach. Additionally, normal spindle configurations and chromosome structures were found in about $60 \%$ of the cases, which was similar and did not differ significantly from those found in follicles from unstimulated control groups. The achieved similar final developmental and maturation outcomes in both groups reflected the efficiency of the method. However, the lower numbers of secondary follicles retrieved from superovulated ovaries meeting the criteria for culture might be a consequence of a successful recruitment of growing follicles using high dose of gonadotropins, and thus fewer secondary follicles were left in these ovaries in comparison with the unstimulated control group.

During culture, follicular growth curves and trends were similar between groups, with slower growth during the first 5 days of culture and faster growth thereafter. Thus, levels of $17 \beta$-estradiol were measured firstly on Day 5 and at two additional timepoints thereafter, including the day of the end of culture. The increasing secretion of $17 \beta$-estradiol showed a correlation with the increase of follicular sizes, as expected [27-29].

It was observed under the culture that follicles from superovulated ovaries seemed to adapt faster to the culture system than control follicles, as indicated by the significantly higher percentages of follicles attached to the culture dish during the initial days of culture. These follicles also grew faster initially, since the start of culture, compared to the control group. A possible explanation is that the gonadotropins previously received in-vivo exerted a continuing effect on the proliferation and function of granulosa cells, reflected also in the higher levels of $17 \beta$-estradiol secreted in the superovulated follicle group vs controls. Apparently, this effect did not influence the final outcomes of the culture, as similar percentages of cultured follicles formed an antrum-like cavity, survived the whole culture and met the selection criteria to be put into IVM in both groups. The timing and follicle selection for IVM were according to the final follicle size achieved on Day 12 of culture $[20,21]$.

The maturation ability of oocytes following hCG-induced IVM was also preserved in the group of follicles retrieved from superovulated ovaries, and similar percentages of MII oocytes were obtained in both groups. Additionally, similar percentages of MII oocytes with normal spindle structure and chromosome configurations were also found, without significant differences between the groups. Our study provides indications that support the retrieval of preantral secondary follicles for in-vitro culture even after COS. This is proposed as a rescue method, and it should be further investigated aiming at finding 
novel fertility preservative methods to women who have failed to produce mature oocytes for cryopreservation after cycle(s) of COS treatment. The method proposed herein could offer a resource for obtaining mature oocytes through collecting the ovarian tissue and performing secondary follicles isolation, in-vitro culture and IVM.

Further development of the method hereby described is needed for the establishment of clinically standardized fertility preservative methods. Use of human materials in experimental research will increase the similarity to the clinical setting instead of using mice. There are established methods for conducting human secondary follicle in-vitro culture and oocytes IVM isolated from human ovarian cortical strips [30,31]. Additional investigation of the fertilization competence of mature oocytes obtained in vitro through this approach should be conducted, and importantly, the follow-up of their offspring.

Author Contributions: Conceptualization, X.H., A.A. and K.A.R.-W.; methodology, X.H., A.A.; validation, X.H., A.A. and K.A.R.-W.; formal analysis, X.H.; investigation, X.H., A.A.; resources, K.A.R.-W.; writing-original draft preparation, X.H. and K.A.R.-W.; writing-review and editing, X.H., A.A. and K.A.R.-W.; visualization, X.H.; supervision, K.A.R.-W.; project administration, K.A.R.-W.; funding acquisition, K.A.R.-W. All authors have read and agreed to the published version of the manuscript.

Funding: This study was funded by the Swedish Childhood Cancer Foundation (PR2016-0115, PR2020-0136), the Swedish Cancer Society (CAN 2017/704, 200170 F), the Swedish Research Council (Dnr 2020-02230), Radiumhemmets Research Funds Grant for clinical researchers 2020-2025, the Stockholm County Council (FoUI-953912) and the Karolinska Institutet Research grants in pediatrics from the Birgitta and Carl-Axel Rydbeck Donation, 2020-00339 to K.A.R.-W. Doctoral candidate X.H. has been supported by the China Scholarship Council.

Institutional Review Board Statement: The study was conducted according to the Animal Protection Law, the Animal Protection Regulation, the Regulation of the Swedish National Board for Laboratory Animals and approved by the Ethics Committee of Karolinska Institutet (03711-2020 and 1372 (2018-01-24)).

Informed Consent Statement: Not applicable.

Acknowledgments: We sincerely thank Raoul Kuiper at the FENO core facility at Karolinska Institutet for excellent technical support in the usage of Confocal systems. We also thank Moustapha Hassan, Sandra Oerther, Jessica Åkerlund, Therése Ahlström and Åsa Eriksson at the experimental research facility PKL5, Novum, Karolinska Institutet, Huddinge for providing superovulated mice ovaries. We thank the Department of Oncology and Pathology of Karolinska Institutet and particularly Professor Jonas Bergh, for administrative support and providing an excellent experimental platform.

Conflicts of Interest: The authors declare no conflict of interest. The funders had no role in the design of the study; in the collection, analyses, or interpretation of data; in the writing of the manuscript, or in the decision to publish the results.

\section{References}

1. World Health Organization. Available online: https://www.who.int/health-topics/cancer\#tab=tab_1 (accessed on 21 January 2021).

2. Miller, K.D.; Nogueira, L.; Mariotto, A.B.; Rowland, J.H.; Yabroff, K.R.; Alfano, C.M.; Jemal, A.; Kramer, J.L.; Siegel, R.L. Cancer treatment and survivorship statistics, 2019. CA Cancer J. Clin. 2019, 69, 363-385. [CrossRef]

3. Duffy, C.; Allen, S. Medical and psychosocial aspects of fertility after cancer. Cancer J. 2009, 15, 27-33. [CrossRef]

4. Abedelahi, A.; Rezaei-Tavirani, M.; Mohammadnejad, D. Fertility preservation among the cancer patients by ovarian tissue cryopreservation, transplantation, and follicular development. Iran. J. Cancer Prev. 2013, 6, 123-132.

5. Rodriguez-Wallberg, K.A.; Oktay, K. Fertility preservation during cancer treatment: Clinical guidelines. Cancer Manag. Res. 2014, 6, 105-117. [PubMed]

6. Donnez, J.; Dolmans, M.M. Fertility preservation in women. Nat. Rev. Endocrinol. 2013, 9, 735-749. [CrossRef]

7. Rodriguez-Wallberg, K.A.; Oktay, K. Options on fertility preservation in female cancer patients. Cancer Treat. Rev. 2012, 38, 354-361. [CrossRef]

8. Rudick, B.; Opper, N.; Paulson, R.; Bendikson, K.; Chung, K. The status of oocyte cryopreservation in the United States. Fertil. Steril. 2010, 94, 2642-2646. [CrossRef] 
9. Cobo, A.; Garrido, N.; Pellicer, A.; Remohí, J. Six years' experience in ovum donation using vitrified oocytes: Report of cumulative outcomes, impact of storage time, and development of a predictive model for oocyte survival rate. Fertil. Steril. 2015, 104, 1426-1434.e1-8. [CrossRef]

10. ESHRE Guideline Group on Female Fertility Preservation; Anderson, R.A.; Amant, F.; Braat, D.; D'Angelo, A.; Chuva de Sousa Lopes, S.M.; Demeestere, I.; Dwek, S.; Frith, L.; Lambertini, M.; et al. ESHRE guideline: Female fertility preservation. Hum. Reprod. Open 2020, 2020, hoaa052.

11. Diaz-Garcia, C.; Domingo, J.; Garcia-Velasco, J.A.; Herraiz, S.; Mirabet, V.; Iniesta, I.; Cobo, A.; Remohí, J.; Pellicer, A. Oocyte vitrification versus ovarian cortex transplantation in fertility preservation for adult women undergoing gonadotoxic treatments: A prospective cohort study. Fertil. Steril. 2018, 109, 478-485.e2. [CrossRef]

12. Pacheco, F.; Oktay, K. Current Success and Efficiency of Autologous Ovarian Transplantation: A Meta-Analysis. Reprod. Sci. 2017, 24, 1111-1120. [CrossRef] [PubMed]

13. Gosden, R.G. Gonadal tissue cryopreservation and transplantation. Reprod. Biomed. Online 2002, 4 (Suppl. 1), 64-67. [CrossRef]

14. Fasano, G.; Dechène, J.; Antonacci, R.; Biramane, J.; Vannin, A.S.; Van Langendonckt, A.; Devreker, F.; Demeestere, I. Outcomes of immature oocytes collected from ovarian tissue for cryopreservation in adult and prepubertal patients. Reprod. Biomed. Online 2017, 34, 575-582. [CrossRef] [PubMed]

15. Nikiforov, D.; Junping, C.; Cadenas, J.; Shukla, V.; Blanshard, R.; Pors, S.E.; Kristensen, S.G.; Macklon, K.T.; Colmorn, L.; Ernst, E.; et al. Improving the maturation rate of human oocytes collected ex vivo during the cryopreservation of ovarian tissue. J. Assist. Reprod. Genet. 2020, 37, 891-904. [CrossRef] [PubMed]

16. De Roo, C.; Lierman, S.; Tilleman, K.; Peynshaert, K.; Braeckmans, K.; Caanen, M.; Lambalk, C.B.; Weyers, S.; T’Sjoen, G.; Cornelissen, R.; et al. Ovarian tissue cryopreservation in female-to-male transgender people: Insights into ovarian histology and physiology after prolonged androgen treatment. Reprod. Biomed. Online 2017, 34, 557-566. [CrossRef]

17. Grynberg, M.; Le Bras, A.M.; Hesters, L.; Gallot, V.; Frydman, N. First birth achieved after fertility preservation using vitrification of in vitro matured oocytes in a woman with breast cancer. Ann. Oncol. 2020, 31, 541-542. [CrossRef]

18. Huober-Zeeb, C.; Lawrenz, B.; Popovici, R.M.; Strowitzki, T.; Germeyer, A.; Stute, P.; von Wolff, M. Improving fertility preservation in cancer: Ovarian tissue cryobanking followed by ovarian stimulation can be efficiently combined. Fertil. Steril. 2011, 95, 342-344. [CrossRef]

19. Anastácio, A.; Waterstone, M.; Hao, X.; Poirot, C.; Rodriguez-Wallberg, K.A. Ovarian Follicles Rescued 3 Days after Cyclophosphamide Treatment in Adolescent Mice: An Experimental Study Aiming at Maximizing Methods for Fertility Preservation through In Vitro Follicle Culture. Int. J. Mol. Sci. 2019, 20, 6190. [CrossRef]

20. Anastácio, A.; Rodriguez-Wallberg, K.A.; Chardonnet, S.; Pionneau, C.; Fédérici, C.; Almeida Santos, T.; Poirot, C. Protein profile of mouse ovarian follicles grown in vitro. Basic Sci. Reprod. Med. 2017, 23, 827-841. [CrossRef]

21. Cortvrindt, R.; Smitz, J.; van Steirteghem, A.C. In-vitro maturation, fertilization and embryo development of immature oocytes from early preantral follicles from prepuberal mice in a simplified culture system. Hum. Reprod. 1996, 11, 2656-2666. [CrossRef]

22. Luan, Y.; Edmonds, M.E.; Woodruff, T.K.; Kim, S.Y. Inhibitors of apoptosis protect the ovarian reserve from cyclophosphamide. J. Endocrinol. 2019, 240, 243. [CrossRef] [PubMed]

23. Winship, A.L.; Carpenter, M.; Griffiths, M.; Hutt, K.J. Vincristine Chemotherapy Induces Atresia of Growing Ovarian Follicles in Mice. Toxicol. Sci. 2019, 169, 43-53. [CrossRef] [PubMed]

24. Haas, J.R.; Christian, P.J.; Hoyer, P.B. Effects of impending ovarian failure induced by 4-vinylcyclohexene diepoxide on fertility in C57BL/ 6 female mice. Comp. Med. 2007, 57, 443-449.

25. Zhu, J.; Cui, W.; Dai, Y.F. Production of inbred offspring by intracytoplasmic sperm injection of oocytes from juvenile female mice. Reprod. Fertil. Dev. 2018, 30, 451-458. [CrossRef] [PubMed]

26. Nelson, J.F.; Gosden, R.G.; Felicio, L.S. Effect of dietary restriction on estrous cyclicity and follicular reserves in aging C57BL/6J mice. Biol. Reprod. 1985, 32, 515-522. [CrossRef]

27. Liu, H.C.; He, Z.; Rosenwaks, Z. Mouse ovarian tissue cryopreservation has only a minor effect on in vitro follicular maturation and gene expression. J. Assist. Reprod. Genet. 2003, 20, 421-431. [CrossRef] [PubMed]

28. Liu, H.C.; He, Z.; Rosenwaks, Z. In vitro culture and in vitro maturation of mouse preantral follicles with recombinant gonadotropins. Fertil. Steril. 2002, 77, 373-383. [CrossRef]

29. Liu, H.C.; He, Z.; Rosenwaks, Z. Correlation of somatic cell steroid secretion and quality of generated oocytes after in-vitro stimulation of mouse follicles. J. Assist. Reprod. Genet. 2006, 23, 191-198. [CrossRef]

30. McLaughlin, M.; Albertini, D.F.; Wallace, W.H.; Anderson, R.A.; Telfer, E.E. Metaphase II oocytes from human unilaminar follicles grown in a multi-step culture system. Basic Sci. Reprod. Med. 2018, 24, 135-142. [CrossRef]

31. Xiao, S.; Zhang, J.; Romero, M.M.; Smith, K.N.; Shea, L.D.; Woodruff, T.K. In vitro follicle growth supports human oocyte meiotic maturation. Sci. Rep. 2015, 5, 17323. [CrossRef] 